\title{
Mismatch Information
}

National Cancer Institute

\section{Source}

National Cancer Institute. Mismatch Information. NCI Thesaurus. Code C44474.

Describes how a reporter varies from its ReporterCharacteristics sequence(s) or how a Feature varies from its Reporter sequence. (caMAGE) 